\title{
Universal prevention of schizophrenia and surrogate endpoints at population level
}

\author{
A. Szöke $\cdot$ J. B. Kirkbride $\cdot$ F. Schürhoff
}

Received: 5 October 2013/Accepted: 19 January 2014/Published online: 2 February 2014

(C) Springer-Verlag Berlin Heidelberg 2014

\begin{abstract}
Despite significant progresses in our knowledge of the risk factors for schizophrenia, we still are several steps short of implementing effective prevention strategies. Universal prevention strategies have several theoretical advantages but their implementation has been limited to date by their costs and lack of methods to assess their efficiency. To overcome this limitation, we suggest, based on research from therapeutic trials at individual level, the use of surrogate endpoints (SEs) at population level. We further suggest that subclinical measures of psychosis at population level are good SEs candidates for assessing universal measures for schizophrenia prevention.
\end{abstract}

Electronic supplementary material The online version of this article (doi:10.1007/s00127-014-0829-9) contains supplementary material, which is available to authorized users.

A. Szöke $(\bowtie) \cdot$ F. Schürhoff

AP-HP, Groupe Hospitalier "Mondor", Pôle de Psychiatrie, Créteil 94000, France

e-mail: andrei.szoke@inserm.fr

A. Szöke · F. Schürhoff

INSERM, U955, Equipe 15, Créteil 94000, France

A. Szöke $\cdot$ F. Schürhoff

Université Paris-Est, Faculté de Médecine, Créteil 94000, France

A. Szöke · F. Schürhoff

Fondation Fondamental, Créteil 94000, France

J. B. Kirkbride

Department of Psychiatry, University of Cambridge, Cambridge, UK

\section{Introduction}

Advances in the epidemiology of schizophrenia have uncovered several individual- and population-level environmental risk factors for the disorder. This has raised hopes of both understanding more about the physiopathology of schizophrenia, and designing effective prevention strategies.

The aim of this paper is to evaluate current capacity to translate these findings into the prevention of schizophrenia, and provide an overview of challenges faced by different approaches. Based on this analysis, we discuss the use of surrogate endpoints, at the population level, as a means to deal with what we consider one the most important obstacle in the implementation of universal preventive measures, i.e. difficulties in assessing their impact.

\section{Current strategies for the prevention of schizophrenia and their limitations}

Selective and indicated strategies

Prevention of schizophrenia has been primarily limited to selective and indicated strategies. These approaches aim at preventing transition to psychosis in individuals identified on the basis of their risk to develop schizophrenia e.g. a family history of psychotic disorder (selective prevention) or of evidence of pre-clinical/prodromal psychotic symptoms (indicated prevention). The success of such services, which include early detection and intervention initiatives, depends on their ability to minimise the false negative and false positive rates.

At present, the number of false negatives, i.e. undetected cases, is high. Data from the PACE (Personal Assessment 
and Crisis Evaluation) clinic in northwestern Melbourneone of the best examples of implementation of indicated/ selective prevention of schizophrenia-suggest that $95 \%$ of the subjects that eventually develop schizophrenia are not detected by the current strategies [1, 7, 17]. The number of false positive is also high. Forty-five to $85 \%$ of the subjects identified as "at-risk" (depending on the definition used in each study) will never develop schizophrenia, even in the absence of any specific preventive strategy [16]. Finally, the efficacy of different measures proposed to prevent the onset of overt psychosis in subjects at risk has not been conclusively established [11].

Universal prevention strategy in schizophrenia and its limitations: the need for efficacy measures

Universal prevention strategies are targeted at whole groups' population, without any explicit attempt to identify people on the basis of increased risk (e.g. adding fluoride to water supplies to reduce tooth decay). Thus, such approaches are not dependent on a screening process. Furthermore, compared to indicated or selective measures, they may result in the prevention of a greater number of cases than indicated or selective strategies. Of direct relevance to mental health, universal strategies may also be less stigmatizing since they target whole population groups, and can have beneficial effects that go beyond the disorder that is the primary goal of prevention.

Many prevention researchers, and particularly those whose focus is on mental health outcomes, consider that despite the potential that universal prevention strategies have, their utility is limited by their costs [15]. Although for a given population, indicated prevention strategies can be implemented at lesser costs than universal strategies, the decision to use one or other of these strategies should ultimately be based on their cost-effectiveness, i.e. resources spent per case prevented, which is presently extremely difficult to demonstrate for schizophrenia. Although there is rich variation in the incidence of disorder, the absolute incidence of such disorders is low, and latency between some exposures and the manifestation of disorder is long. For these reasons, demonstration of efficacy of any universal prevention strategy by assessing impact on the incidence of schizophrenia would be, if at all feasible, long and expensive.

\section{Surrogate endpoints and related concepts at individual and population level}

Schizophrenia is not the only field to face such challenges. Researchers involved in pharmacological/therapeutic studies in which the number of outcome events is very small (or where the time to outcome is very long) are faced with similar problems. Examples include trials of new treatments for HIV infection, for stroke prevention or for chronic diseases such as diabetes. In these situations, one viable solution is to assess the impact of the intervention using surrogate endpoints (SEs): i.e. biomarkers that are more convenient to measure and, as they are linked to the outcome of interest, can be used as a substitute for it.

In this paper, we argue that to evaluate the impact of universal prevention strategies on the incidence of schizophrenia, a similar approach, at the population level, could be useful.

The need for validated surrogate endpoints at the population level

This approach has already been used (although not explicitly) in the evaluation of universal prevention strategies in other mental health domains. For example, Shochet et al. [10] evaluated the efficacy of preventive measures for depression in secondary school students by measuring levels of depressive symptomatology and hopelessness. However, because the use of those SEs has not been preceded by a convincing evaluation of their link with the measure of interest, in this case clinical depression, the results of those studies have been considered with scepticism. For example, in a meta-analysis on prevention measures for depression, Cuijpers et al. [3] explicitly excluded all studies that used outcome measures other than a diagnosis of depression. This suggests that before using SEs for prevention studies, at the population level, an explicit and rigorous approach similar to that used in the development and validation of SEs for clinical trials, needs to be applied.

\section{Definitions and general framework for SEs} from clinical trials

A clinical trial measures the effect of a therapeutic intervention on a target, which reflects the principal outcome we are interested in, i.e. patient survival, function or wellbeing. This target is referred to as a clinical endpoint (CE).

Surrogate endpoints are biomarkers that predict the effect of a therapeutic intervention on the CE, and could thus be used as substitutes when the CE is difficult or impractical to measure (Fig. 1). Sometimes the SE, although not the central outcome measure (i.e. the $\mathrm{CE}$ ), also reflect a real clinical benefit. To signify this added quality, such SEs are called intermediate clinical endpoints (ICE).

An example of an SE is the use of CD4 counts, instead of survival rates (CE) in people with HIV infection, in clinical trials of antiretroviral drugs. Another possible SE 


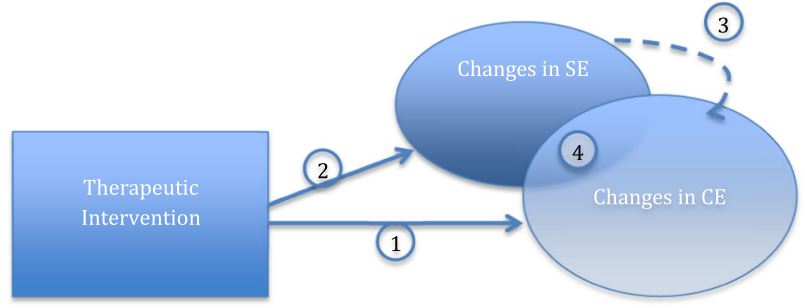

Fig. 1 Conceptual overview of surrogate endpoints. A successful therapeutic intervention leads to changes in $\mathrm{CE}$ (1) and changes in SE (2). As figured with the dotted arrow (3) some of the changes in CE could be (but are not necessarily) the consequence of changes in SE. Changes in SE reflect a fraction of changes in CE (surrogacy) represented by the common area (4)

in this case could be the number of opportunistic infections. Because this later measure also reflects a clinical benefit (fewer opportunistic infections being in itself a desirable outcome), it could be considered as an ICE.

Surrogate endpoints provide some tangible benefits for the development of therapeutic interventions. A good SE will save research time and money and, more importantly, could save lives or avoid suffering by speeding up the process of treatment approval. However, before using a SE, a thorough evaluation of the proposed SE is needed [6].

\section{Surrogate endpoints validation}

Two characteristics are essential for a SE, both of them relative to the CE: surrogacy (i.e. SEs have to predict the effect of the treatment on $\mathrm{CE}$ ) and convenience (i.e. be more easy to measure than the $\mathrm{CE}$ ).

In the first stages, putative SEs are chosen based on their plausibility i.e. they are reasonably likely, based on existing scientific evidence to predict the CE. Most arguments at this stage come from epidemiological studies that suggest a statistical relationship between the SE and CE in basic conditions, i.e. before any therapeutic intervention, or from pathophysiological studies, or causal models that suggest that the SE is in the disease's pathway. A measure will be used as a putative SE, keeping in mind the risk of false results, until definite demonstration of its validity, i.e. proof of its surrogacy.

As pointed by Lesko and Atkinson [6], the level of stringency required for a SE depends on its intended use and although validated SEs are needed when they are used for final approval of a new treatment, putative SEs can be useful in the first phases of drug development, for example to provide proof of concept.

Since SEs are used to record treatment effects with less expenditure and at a faster pace, they must also be more convenient to measure than the CE. Boissel et al. [2] argued that the most important characteristic a convenient
SE must possess is frequency, i.e. it should occur more often than the corresponding $\mathrm{CE}$, thus limiting the time needed to assess the outcome. A particularly favourable case is the use of quantitative, continuous variables as $\mathrm{SE}$ of binary $\mathrm{CE}$, which enhances statistical power and reduces time needed to perform trials [2].

Finally, to be useful for assessing the effect of a treatment, the measures used as SEs must be modifiable [5] and reliable [6].

We suggest that conceptually similar criteria may be useful for identifying putative SEs at population level, for public health interventions.

However, translation of concepts from the individual to the population level has to be done with caution. Epidemiologists are well aware of the risk of serious errors when inferences are not confined at the level of observation individual vs. population - the so-called atomistic fallacy. A similar situation could arise when considering that good SEs for clinical trials would automatically make good SEs for population interventions. Thus, for any SE suggestions have to be made and arguments examined at the level (individual vs. population) of interest.

\section{Putative SEs at population level for assessing the efficacy of preventive measures for schizophrenia}

In this section, we consider the use of SEs in studies designed to assess the impact of putative population-level prevention strategies for the incidence of psychotic disorders. We will discuss two measures of the extended psychotic phenotype which we suggest may be suitable candidate SEs in studies of universal prevention strategies for schizophrenia: the prevalence of psychotic-like experiences (PLEs) and population mean of psychometric schizotypy (PS) based on a quantitative assessment of dimensional schizotypy [4], presumed to lie on a continuum in the general population.

As pointed before, their plausibility and convenience will be examined at population level. The fact that similar measures at individual level are or are not associated with schizophrenia and/or more convenient than the diagnosis of schizophrenia is not relevant for this discussion.

\section{Plausibility}

The theoretical model on which we base our suggestion is the continuum theory of psychosis. According to this theory, schizophrenia is at one end of a continuum of psychotic experiences, most of which lays in the normal range and do not warrant clinical intervention. At the population level, this theory suggests that, together with clinical cases of psychosis there are more frequent cases of an "extended 
phenotype" characterized by sub-threshold manifestations (be it in number of symptoms i.e. PLE or in intensity i.e. schizotypy) [13].

Several direct or indirect epidemiological arguments support the use of these measures as plausible SEs. First, for other medical or psychiatric conditions, a link between the number of deviant (pathologic) subjects in a population and mean population measures of related physiological measures (i.e. in the normal range) has been observed. For example, Rose and Day [9] showed, based on the analysis of multiple independent population samples that the number of subjects suffering from hypertension correlates to mean population measures of blood pressure. Whittington and Huppert [14] applied the same approach to mental health, showing that changes in the mean level of psychiatric symptoms in the community, measured by the General Health Questionnaire, were linked to changes over time in the prevalence of psychiatric disorders.

Second, population-based epidemiological studies have shown that risk factors for schizophrenia (e.g. cannabis consumption, minority status, urbanicity) are associated with increases in the measures of the extended phenotype (e.g. [8]).

Finally, these indirect arguments are complemented by data showing that, across different populations, there is a strong link between the level of psychotic-like symptoms and number of cases of clinical psychosis [12].

\section{Convenience}

Measuring the incidence of schizophrenia in a population is a difficult process necessitating a complex methodology for identifying all potential cases and then strict procedures to confirm caseness using research criteria and experienced clinicians. Furthermore, because of the relatively low incidence of the disorder, obtaining a precise estimate is a long process - taking place usually over several years.

By contrast, estimating PS or PLEs in a population necessitate self-administered questionnaires or short interviews that do not require researchers with extensive clinical experience. Given that PLEs are far more frequent than schizophrenia [8] and PS is a continuous measure, thought to lie on a continuum in the general population, values can be obtained based on representative samples that could be selected and assessed over shorter intervals.

Although at individual level, measures for PS and PLE, have been proved to be reliable and can change over time, this does not automatically ensure reliability and/or capacity to change for the same measures at population level. Thus, specific exploration of these properties, at the population level is required.

Table 1 (supplementary material available on line) provides a summary and comparison of PLEs and PS at population level with a classic SE at individual level (blood pressure as SE for stroke).

\section{Discussion}

Several questions are still to be answered regarding how this proposal could best be used in the specific case of schizophrenia prevention.

A first question is about the choice of the CE of interest. Although in this paper, for simplicity's sake, we used the example of schizophrenia we are aware that there are also arguments for using a more large "psychosis" category and even include affective disorders with psychotic features.

The best population SE measure is still to be found. Two measures have been suggested in this paper (PS and PLEs) but other potential measures may be more suited. For example, given the relatively narrow window for the age of onset in schizophrenia, restricting the measures only to a sub-population of young people could prove to be a better SE for the incidence of schizophrenia. Thus, the choice of the best SE will probably wait for further studies. If studies are implemented at this stage, it will be wiser, when possible, to plan and test for multiple alternative definitions and measures for the SEs and CEs.

Also, it would be of interest to choose the SE as a potential ICE. Thus, if reducing the risk factors (RF) leads to reduction in the ICE but not in the CE (surrogacy not confirmed) there would still be a benefit. PS could well be an ICE as high levels of schizotypy have been associated with suffering, lower quality of life and risk for severe comorbidities or complications. For similar reasons, when possible, it would be better to choose the RF among factors that, if efficiently addressed, will lead to benefits beyond what is expected from preventing the CE (e.g. cannabis) and to take into consideration all the outcomes associated with the intervention when assessing the global cost-benefit of the intervention.

An illustration of how research based on the ideas discussed here could be implemented is presented in supplementary material S2.

\section{Conclusion}

Several theoretical and practical steps are needed before the use of SE at population level could be effective. Given the potential of universal measures for schizophrenia prevention, if the strategy proposed here could assist the finding and implementation of efficient interventions the efforts needed to overcome the present limitations could be worthwhile.

Conflict of interest The authors declare that they have no conflict of interest. 


\section{References}

1. Amminger GP, Harris MG, Conus P, Lambert M, Elkins KS, Yuen HP, McGorry PD (2006) Treated incidence of first-episode psychosis in the catchment area of EPPIC between 1997 and 2000. Acta Psychiatr Scand 114:337-345

2. Boissel JP, Collet JP, Moleur P, Haugh M (1992) Surrogate endpoints: a basis for a rational approach. Eur J Clin Pharmacol 43:235-244

3. Cuijpers P, Van Straten A, Smit F (2005) Preventing the incidence of new cases of mental disorders: a meta-analytic review. J Nerv Mental Dis 193:119-125

4. Green MJ, Boyle GJ, Raine A (2008) Schizotypal personality models (Chapter 19). In: Boyle GJ, Matthews G, Saklofske DH (eds) The SAGE handbook of personality theory and assessment: Vol 1, Personality theories and models. SAGE Publications Ltd, London

5. Lassere MN, Johnson KR, Boers M, Tugwell P, Brooks P, Simon L, Strand V, Conaghan PG, Ostergaard M, Maksymowych WP, Landewe R, Bresnihan B, Tak PP, Wakefield R, Mease P, Bingham CO 3rd, Hughes M, Altman D, Buyse M, Galbraith S, Wells G (2007) Definitions and validation criteria for biomarkers and surrogate endpoints: development and testing of a quantitative hierarchical levels of evidence schema. J Rheumatol 34:607-615

6. Lesko LJ, Atkinson AJ Jr (2001) Use of biomarkers and surrogate endpoints in drug development and regulatory decision making: criteria, validation, strategies. Annu Rev Pharmacol Toxicol 41:347-366

7. Lin A. (2010) Why Transition is not the whole story: neurocognitive function and risk for psychosis. Thesis for the degree of Doctor of Philosophy. Department of Psychology. The University of Melbourne

8. Linscott RJ, van Os J (2013) An updated and conservative systematic review and meta-analysis of epidemiological evidence on psychotic experiences in children and adults: on the pathway from proneness to persistence to dimensional expression across mental disorders. Psychol Med 43(6):1133-1149

9. Rose G, Day S (1990) The population mean predicts the number of deviant individuals. Br Med J 301:1031-1034

10. Shochet IM, Dadds MR, Holland D, Whitefield K, Harnett PH, Osgarby SM (2001) The efficacy of a universal school-based program to prevent adolescent depression. Journal of Clinical Child Psychology 30:303-315

11. Stafford MR, Jackson H, Mayo-Wilson E, Morrison AP, Kendall T (2013) Early interventions to prevent psychosis: systematic review and meta-analysis. BMJ 346:f185. doi:10.1136/bmj.f185

12. van Os J, Hanssen M, Bijl RV, Vollebergh W (2001) Prevalence of psychotic disorder and community level of psychotic symptoms: an urban-rural comparison. Arch Gen Psychiatry 58:663-668

13. van Os J, Linscott RJ, Myin-Germeys I, Delespaul P, Krabbendam L (2009) A systematic review and meta-analysis of the psychosis continuum: evidence for a psychosis proneness-persistence-impairment model of psychotic disorder. Psychol Med 39:179-195

14. Whittington JE, Huppert FA (1996) Changes in the prevalence of psychiatric disorder in a community are related to changes in the mean level of psychiatric symptoms. Psychol Med 26:1253-1260

15. Yung AR, Killackey E, Hetrick SE, Parker AG, Schultze-Lutter F, Klosterkoetter J, Purcell R, Mcgorry PD (2007) The prevention of schizophrenia. Int Rev Psychiatry 19:633-646

16. Yung AR, Yuen HP, Berger G, Francey S, Hung TC, Nelson B, Phillips L, McGorry P (2007) Declining transition rate in ultra high risk (prodromal) services: dilution or reduction of risk? Schizophr Bull 33:673-681

17. Yung AR, Nelson B, Stanford C, Simmons MB, Cosgrave EM, Killackey E, Phillips LJ, Bechdolf A, Buckby J, McGorry PD (2008) Validation of "prodromal" criteria to detect individuals at ultra high risk of psychosis: 2 year follow-up. Schizophr Res 105:10-17 